\title{
Improved Detection of Sleeping Sickness Cases by LED Fluorescence Microscopy: Evidence from a Prospective Multi-Centric Study in the Democratic Republic of the Congo
}

\author{
Patrick Mitashi', Pascal Lutumba1, Crispin Lumbala², Paul Bessell³, Sylvain Biéler ${ }^{3 *}$, \\ Joseph Mathu Ndung' $\mathbf{u}^{3}$ \\ ${ }^{1}$ Tropical Medicine Department, Faculty of Medicine, University of Kinshasa, Kinshasa, Democratic Republic of \\ the Congo \\ ${ }^{2}$ Programme National de Lutte contre la Trypanosomiase Humaine Africaine (PNLTHA), Croisement de l'Avenue \\ de la Libération et du Boulevard Triomphal 1, Kinshasa, Democratic Republic of the Congo \\ ${ }^{3}$ Foundation for Innovative New Diagnostics (FIND), Campus Biotech, Chemin des Mines 9, Geneva, Switzerland \\ Email: drmitashi@yahoo.fr, pascal lutumba@yahoo.fr, crispinlumbala@gmail.com, \\ paul.bessell@finddiagnostics.org, sylvain.bieler@finddiagnostics.org, joseph.ndungu@finddiagnostics.org
}

Received 20 January 2015; accepted 27 March 2015; published 3 April 2015

Copyright (C) 2015 by authors and Scientific Research Publishing Inc.

This work is licensed under the Creative Commons Attribution International License (CC BY). http://creativecommons.org/licenses/by/4.0/

c) (i) Open Access

\begin{abstract}
Background: Confirmatory diagnosis of Trypanosoma brucei gambiense human African trypanosomiasis (HAT) is based on demonstration of parasites by microscopy. However, the sensitivity of routine microscopy methods is very low, and many cases are missed and left untreated. A clinical study was conducted in the Democratic Republic of the Congo to evaluate the accuracy of improved microscopy methods in diagnosis of HAT. These included examination by fluorescence microscopy (FM) of acridine orange (AO) stained smears of whole blood and smears made following a new procedure for concentrating trypanosomes by selective lysis of red blood cells (RBC). Methodology/Principal Findings: Venous blood was collected from 213 HAT cases, 101 HAT suspects and 95 controls and used to determine the accuracy of four microscopy methods: bright field microscopy of Giemsa-stained thick blood smears, FM of AO-stained thick blood smears, FM of AO-stained thick blood smears prepared after RBC lysis and concentration, and FM of AO-stained thin blood smears prepared after RBC lysis and concentration. The sensitivity of FM using thick blood smears stained with AO was 3 times higher than bright field microscopy using Giemsa-stained thick blood smears
\end{abstract}

*Corresponding author.

How to cite this paper: Mitashi, P., et al. (2015) Improved Detection of Sleeping Sickness Cases by LED Fluorescence Microscopy: Evidence from a Prospective Multi-Centric Study in the Democratic Republic of the Congo. Microscopy Research, 3, 17-25. http://dx.doi.org/10.4236/mr.2015.32003 
[19.7\% (95\% CI: $14.9 \%$ - 25.6\%) versus $6.1 \%$ (95\% CI: 3.6\% - 10.2\%)]. When the RBC lysis and concentration procedure was included, sensitivity of the test was further enhanced to $23.0 \%$ (95\% CI: $17.9 \%-29.1 \%$ ) with thick blood smears and $34.3 \%$ (95\% CI: $28.2 \%-40.9 \%$ ) with thin blood smears. Specificity of all four microscopy methods was 100\% (95\% CI: $96.1 \%-100.0 \%)$. However, the miniature anion exchange chromatography technique (mAECT) and capillary tube centrifugation (CTC) method remained more sensitive. Conclusions: These new methods have practical advantages, including shorter staining time, ease of demonstration of parasites, and the possibility of archiving slides. They could, therefore, be alternative methods to improve case detection where concentration procedures such as MAECT or CTC are not performed.

\title{
Keywords
}

\author{
Human African Trypanosomiasis, Trypanosome, LED Fluorescence Microscopy, Red Blood Cell \\ Lysis, Diagnosis
}

\section{Introduction}

Human African trypanosomiasis (HAT), or sleeping sickness, is a vector-borne disease caused by the protozoan parasites Trypanosoma brucei (T.b.) gambiense and T.b. rhodesiense. It mainly affects impoverished rural communities in sub-Saharan Africa, where health systems are weak. Both subspecies are cyclically transmitted by infected tsetse flies of the Glossina genus [1]. The chronic form of sleeping sickness is caused by T.b. gambiense in western and central Africa and is responsible for the majority of HAT cases, while T.b. rhodesiense causes a more fulminant, acute form in eastern and southern Africa. In recent years, the number of new cases of HAT reported to WHO has decreased dramatically from 25,945 in 2000 to less than 10,000 in 2009 [2]. This trend was maintained in 2012 with 7197 new cases reported [3]. However, the actual number of patients with sleeping sickness is estimated to be much higher, as many cases remain undiagnosed or unreported [4].

Sleeping sickness is almost invariably fatal if left untreated and progressed in two stages [5]. The haemolymphatic stage (first stage) is characterized by fever, headache and joint pains. In the second stage, or neurological stage, parasites cross the blood-brain barrier and invade the central nervous system (CNS) [6]. In the absence of treatment, patients develop neuropsychiatric symptoms, such as mental disturbances, neurological disorders, disruption of the sleep-wake cycle, coma and death [7]-[9]. Treatment of stage 2 patients is more difficult and although curative therapy is available, some patients are left with permanent sequellae. Detection of infected individuals in the first stage is therefore critical, and highlights the need for sensitive and specific diagnostic tools.

While the prevalence of T.b. gambiense HAT has been declining, interruption of control efforts will inevitably result in resurgence [10]. When the incidence of HAT is low, active screening is uneconomical, and national control programmes are encouraged to integrate control and surveillance activities in the general healthcare system [11]. However, some of the diagnostic tests for HAT that are currently available are not compatible with the poor infrastructure that is found in primary healthcare facilities, especially in rural areas, hindering the participation of such facilities in effective control and surveillance of the disease [12]. The concentration of T.b. gambiense parasites in blood is usually very low, which makes direct microscopy notoriously insensitive. Microscopy has to therefore be combined with prior parasite concentration methods, such as the Woo or capillary tube centrifugation (CTC) technique and the minicolumn or miniature anion exchange centrifugation technique (mAECT) [13]. Even with these concentration methods, up to $30 \%$ of cases are still missed, remaining an undetected reservoir for transmission [14]. Confirmatory diagnostic tests that are more sensitive, reliable, affordable and easy to perform in basic health care facilities are therefore needed [15]. Light-emitting diode (LED) fluorescence microscopes, such as the Primo Star iLED that was jointly developed by Carl Zeiss and FIND, are among the few new diagnostic tools that can be considered for use in peripheral healthcare facilities. Recent laboratory studies have shown that combination of LED fluorescence microscopy (LED FM) with concentration of parasites by centrifugation after selective lysis of red blood cells (RBC) followed by staining with acridine orange (AO), improves the detection of T.b. brucei [16]. LED FM could thus be a good candidate to enhance diagnosis of HAT.

The objective of this study was to evaluate the performance of LED FM and AO staining, with or without RBC 
lysis, to diagnose HAT in health centres in comparison with methods that are in current use.

\section{Materials and Methods}

\subsection{Study Area and Enrolment of Participants}

The study was conducted in the East Kasai province of the Democratic Republic of the Congo (DRC). Participants were enrolled at three health centres (Katanda, Tshibila and Ngandajika) and by two mobile teams (Tshilenge and Ngandajika) using routine diagnostic protocols as prescribed by the national HAT control programme (PNLTHA) of DRC. Enrolment was from 28th December 2010 to 9th January 2012. Technicians performing the reference standard and index diagnostic tests were personnel of the PNLTHA who had all been extensively trained on relevant methods. Mobile teams and health centres included two technicians who were dedicated to performing diagnostic tests. The card agglutination test for trypanosomiasis (CATT) was performed on whole blood to screen participants; those testing positive with CATT were subjected to a series of confirmatory tests, including lymph node puncture (in case of swollen cervical nodes), examination of a thick blood smear by microscopy, CTC and mAECT [17] [18]. Tests were performed in sequence and once parasitological confirmation was obtained no further confirmatory tests were carried out. A composite reference standard was used to assess sensitivity and specificity of diagnostic methods. This composite reference standard was negative for a subject if no trypanosomes were found either in lymph node aspirate or in blood by routine parasitology methods and positive if trypanosomes were observed in lymph node aspirate or in blood by any of these routine diagnostic tests. A patient was classified as a HAT case if the composite reference standard was positive. Patients in both early (haemolymphatic) and late (neurological) stages were enrolled. Participants were classified as endemic controls if they had no history of HAT and were negative by CATT on whole blood. HAT suspects were defined as those negative for trypanosomes in lymph node aspirate (if available) and in blood using conventional bright field microscopy methods, including CTC and MAECT, but positive with the CATT test at a dilution of 1:8 or more.

\subsection{Laboratory Tests}

Twelve $\mathrm{ml}$ of venous blood was collected from all participants and used to prepare the following four types of microscopy slides:

1) Thick blood smear ( $5 \mu$ l) stained with Giemsa for examination by bright field microscopy (TBS-Giemsa);

2) Thick blood smear $(5 \mu \mathrm{l})$ stained with acridine orange for examination by fluorescence microscopy (TBS$\mathrm{AO}$ );

3) Thick blood smear $(20 \mu \mathrm{l})$ prepared after red blood cell lysis and concentration, stained with acridine orange for examination by fluorescence microscopy (Lysis-TBS-AO);

4) Thin blood smear $(20 \mu \mathrm{l})$ prepared after red blood cell lysis and concentration, stained with acridine orange for examination by fluorescence microscopy (Lysis-tBS-AO).

Red blood cell lysis and concentration was performed as described previously [16], except that 3 tubes containing $3 \mathrm{ml}$ of venous blood each were used for lysis, and that each pellet was resuspended in $50 \mu$, and pooled to obtain a total volume of $150 \mu \mathrm{l}$ of suspension. Staining of slides with Giemsa and with acridine orange, as well as examination of slides using the Primo Star iLED microscope (Carl Zeiss, GmbH) were also performed as described previously [16].

Using this same sample of venous blood, CTC and mAECT were performed at this stage, as per the routine procedures of the PNLTHA, using 4 capillary tubes of $50 \mu \mathrm{l}$ and $350 \mu \mathrm{l}$ of blood, respectively. All samples were blinded using identification codes and the laboratory personnel had no access to clinical information about study participants or results of previous tests. The number of participants at each site was usually very low. Microscopy slides were therefore stored and read once a week in order to prevent a potential bias that could have been introduced if laboratory technicians knew parasitology results when reading slides on the same day, in spite of blinding.

\subsection{Statistical Methods}

Sensitivity and specificity values and their $95 \%$ confidence intervals were calculated for the tests under investigation in the confirmed HAT patients and in endemic controls, respectively. Sensitivity was defined as the pro- 
portion of positives among confirmed HAT patients diagnosed through routine procedures and specificity as the proportion of controls that tested negative. The sensitivity of various diagnostic algorithms comprising several methods was also analyzed. The result of an algorithm was positive if any or several methods gave a positive result, and it was negative if all methods were negative. Sensitivity and specificity values were calculated with $95 \%$ confidence intervals using binomial Wilson methods [19]. The significance of differences in sensitivity and specificity between two methods or algorithms was evaluated with Pearson's Chi-squared test [20]. Statistical analysis was performed using Stata 11 (Stata Corp., College Station TX, USA) and in the R statistical environment [21].

\subsection{Ethical Considerations}

The protocol for this study was approved by the Ethics Committee of the Public Health School of the University of Kinshasa, DRC. Written informed consent was obtained from each participant before enrolment in the study. HAT patients who were identified during the study were treated in accordance with the standard protocols of the PNLTHA.

\section{Results}

The participants enrolled in the study included 213 HAT cases, 101 suspects and 95 controls (Table 1). Results of the composite reference standard and index test were obtained for all participants. HAT cases included 83 stage 1 patients, 128 stage 2 patients and 2 patients without any information on disease stage. The mean age of participants was 29.8, 31.1 and 33.9 years for cases, suspects and controls, respectively. The male-female gender ratio was $1.32,0.91$ and 0.42 for cases, suspects and controls, respectively. Most frequent symptoms were malaise (29.1\%), fever (24.4\%), headache (16.0\%) and weight loss (6.1\%) among cases, malaise (14.9\%), headache $(13.9 \%)$, fever (10.9\%) and weight loss $(5.9 \%)$ among suspects, and fever $(13.7 \%)$, malaise $(3.2 \%)$, headache $(2.1 \%)$ and weight loss $(2.1 \%)$ among controls. No adverse events from performing the reference or index tests were reported during this study.

Figure 1 shows that staining a thick blood smear with acridine orange and examining it under fluorescent light was more sensitive (19.7\%; 95\% CI: 14.9\% - 25.6\%) than classical Giemsa-stained thick smear examined by bright field microscopy $(6.1 \%$; 95\% CI: 3.6\% - 10.2\%). Sensitivity was further enhanced when a red blood cell lysis and concentration step was included prior to preparing the blood smear and staining it with acridine orange, to $23.0 \%$ (95\% CI: $17.9 \%$ - 29.1\%) with thick blood smears and to $34.3 \%$ (95\% CI: $28.2 \%$ - $40.9 \%$ ) with thin blood smears. However, mAECT and CTC remained the most sensitive techniques at $68.1 \%$ (95\% CI: $61.5 \%-74.0 \%)$ and $52.1 \%(45.4 \%-58.7 \%)$, respectively.

All smear microscopy methods (TBS-Giemsa, TBS-AO, Lysis-TBS-AO and Lysis-tBS-AO) had an excellent specificity (100\%; 95\% CI: 96.1\% - 100.0\%), as no false positive result was reported with any of them (Figure $1(b))$.

None of the 101 HAT suspects enrolled gave a positive result using any of the four smear microscopy methods under investigation, and they were all found to be consistently negative by CTC and by MAECT.

Table 1. Test results in each category of participants. The number of participants with positive and negative results using each test is shown for cases, suspects and controls.

\begin{tabular}{|c|c|c|c|c|c|c|}
\hline \multirow[b]{2}{*}{ Test method } & \multicolumn{2}{|c|}{ Cases $(n=213)$} & \multicolumn{2}{|c|}{ Suspects $(\mathrm{n}=101)$} & \multicolumn{2}{|c|}{ Controls $(n=95)$} \\
\hline & Positive & Negative & Positive & Negative & Positive & Negative \\
\hline mAECT & 145 & 68 & 0 & 101 & 1 & 94 \\
\hline CTC & 111 & 102 & 0 & 101 & 1 & 94 \\
\hline Lysis-tBS-AO & 73 & 140 & 0 & 101 & 0 & 95 \\
\hline Lysis-TBS-AO & 49 & 164 & 0 & 101 & 0 & 95 \\
\hline TBS-AO & 42 & 171 & 0 & 101 & 0 & 95 \\
\hline TBS-Giemsa & 13 & 200 & 0 & 101 & 0 & 95 \\
\hline
\end{tabular}



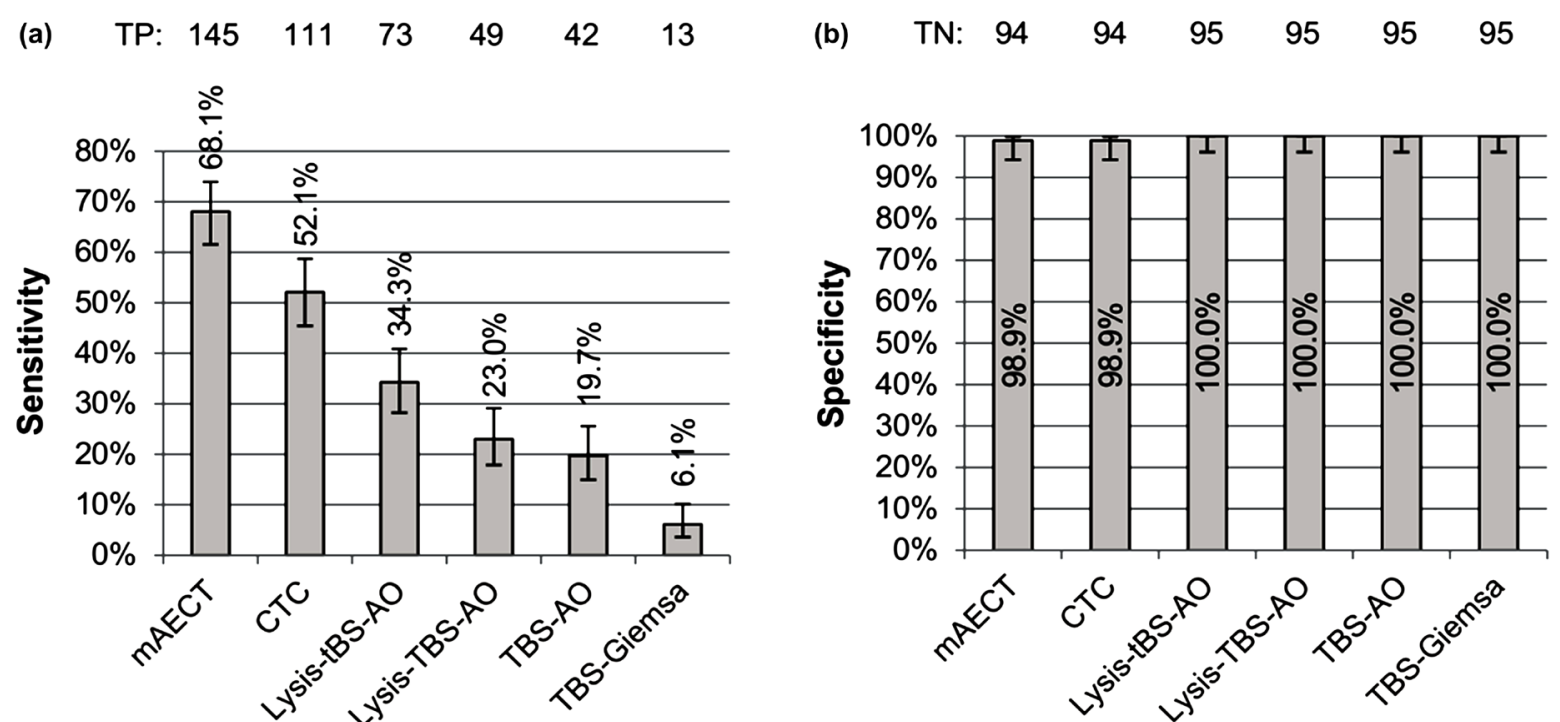

Figure 1. Sensitivity (a) and specificity (b) of various methods in demonstrating trypanosomes in the blood of HAT patients. The number of cases correctly identified with each method (TP, true positives) and the number of controls correctly identified with each method (TN: true negatives) are shown above the graphs. mAECT: miniature anion exchange centrifugation technique; CTC: capillary tube centrifugation; Lysis-tBS-AO: thin blood smear $(20 \mu 1)$ prepared after red blood cell lysis and concentration, stained with acridine orange and examined by fluorescence microscopy; Lysis-TBS-AO: thick blood smear $(20 \mu \mathrm{l})$ prepared after red blood cell lysis and concentration, stained with acridine orange and examined by fluorescence microscopy; TBS-AO: thick blood smear $(5 \mu \mathrm{l})$ stained with acridine orange and examined by fluorescence microscopy; TBSGiemsa: thick blood smear $(5 \mu 1)$ stained with Giemsa and examined by bright field microscopy. Error bars indicate $95 \%$ confidence intervals.

Since smear microscopy is often used in combination with other tests in order to enhance case detection, the sensitivity of various diagnostic algorithms was also calculated. Figure 2 shows that examination of thick smears stained with acridine orange in combination with CTC (CTC, TBS-AO) resulted in 117 HAT cases being detected, while CTC combined with thick smears stained with Giemsa (CTC, TBS-Giemsa) detected 112 cases. However, the difference in sensitivity between these two algorithms was not significant $(P=0.698)$. Similarly, when an algorithm of thin smears prepared using the RBC lysis and concentration procedure and stained with acridine orange combined with CTC (CTC, Lysis-tBS-AO) was used, the number of HAT cases detected increased to 119 , but the corresponding sensitivity was not significantly higher than when using an algorithm combining CTC and TBS-Giemsa $(\mathrm{P}=0.560)$.

The sensitivity values of diagnostic algorithms that include both CTC and MAECT in addition to each of the four smear microscopy methods under investigation are shown in Figure 3. Again, there was no significant difference in sensitivity between any of these algorithms $(\mathrm{P}>0.9)$.

Finally, an analysis of algorithms that include examination of lymph node aspirate (LN) in addition to other tests was performed. Figure 4(a) shows that combining LN with acridine orange thick smear fluorescence microscopy gave a sensitivity of $47.9 \%$ (95\% CI: $41.3 \%-54.6 \%)$, while the sensitivity of LN combined with Giemsa thick smear bright field microscopy was only $36.6 \%$ (95\% CI: $30.4 \%-43.3 \%$ ). When CTC was added to these algorithms (Figure 4(b)), sensitivity values were improved further. For example, when acridine orange thick smear fluorescence microscopy was combined with LN and CTC, a sensitivity of $67.1 \%$ (95\% CI: $60.6 \%$ $73.1 \%$ ) was achieved.

\section{Discussion}

This study has shown that fluorescence microscopy of thick blood smears stained with acridine orange is at least 3 times more sensitive than routine bright field microscopy of slides stained with Giemsa, while specificity remains unaltered at $100 \%$. The number of HAT cases detected when this new method is combined with other diagnostic tests such as LN or CTC is higher, when compared with Giemsa, although this effect becomes less marked. 


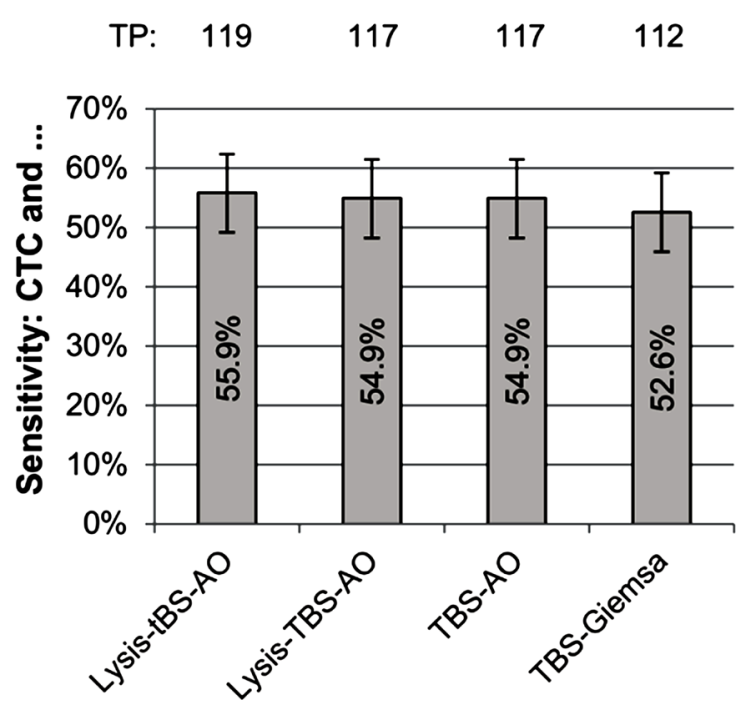

Figure 2. Sensitivity of diagnostic algorithms for HAT in which capillary tube centrifugation (CTC) is combined with a smear microscopy method. An algorithm is positive if either or both CTC and the smear microscopy method give a positive result. The number of HAT cases correctly identified with each algorithm (TP: true positives) is shown above the graph. Lysis-tBS-AO: thin blood smear $(20 \mu \mathrm{l})$ prepared after red blood cell lysis and concentration, stained with acridine orange and examined by fluorescence microscopy; Lysis-TBS-AO: thick blood smear $(20 \mu \mathrm{l})$ prepared after red blood cell lysis and concentration, stained with acridine orange and examined by fluorescence microscopy; TBS-AO: thick blood smear $(5 \mu \mathrm{l})$ stained with acridine orange and examined by fluorescence microscopy; TBSGiemsa: thick blood smear $(5 \mu \mathrm{l})$ stained with Giemsa and examined by bright field microscopy. Error bars indicate $95 \%$ confidence intervals.

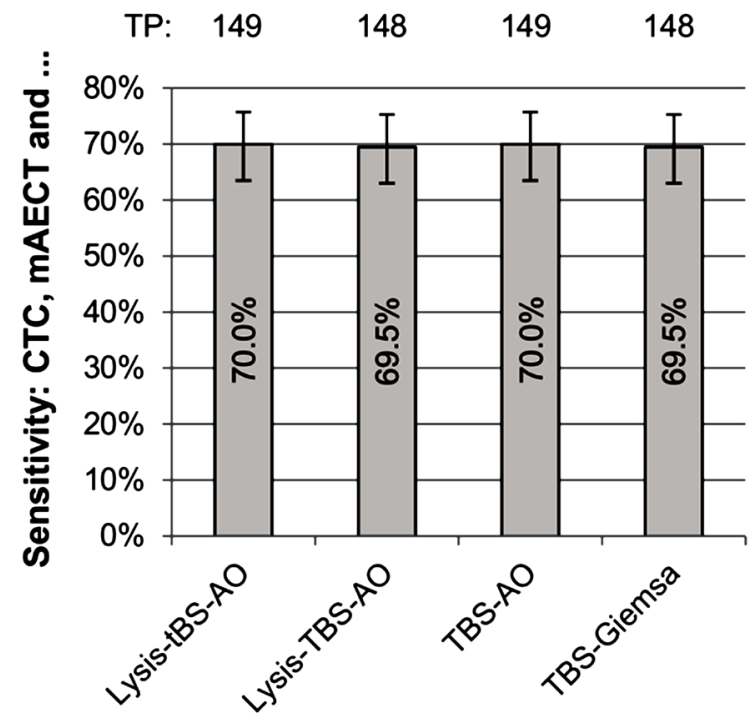

Figure 3. Sensitivity of diagnostic algorithms for HAT in which capillary tube centrifugation (CTC) and the miniature anion exchange centrifugation technique (mAECT) are combined with a smear microscopy method. The result of an algorithm is positive if any or several of the methods give a positive result. The number of HAT cases correctly identified with each algorithm (TP: true positives) is shown above the graph. Lysis-tBS-AO: thin blood smear $(20 \mu \mathrm{l})$ prepared after red blood cell lysis and concentration, stained with acridine orange and examined by fluorescence microscopy; Lysis-TBS-AO: thick blood smear $(20 \mu \mathrm{l})$ prepared after red blood cell lysis and concentration, stained with acridine orange and examined by fluorescence microscopy; TBS-AO: thick blood smear $(5 \mu 1)$ stained with acridine orange and examined by fluorescence microscopy; TBS-Giemsa: thick blood smear $(5 \mu \mathrm{l})$ stained with Giemsa and examined by bright field microscopy. Error bars indicate $95 \%$ confidence intervals. 

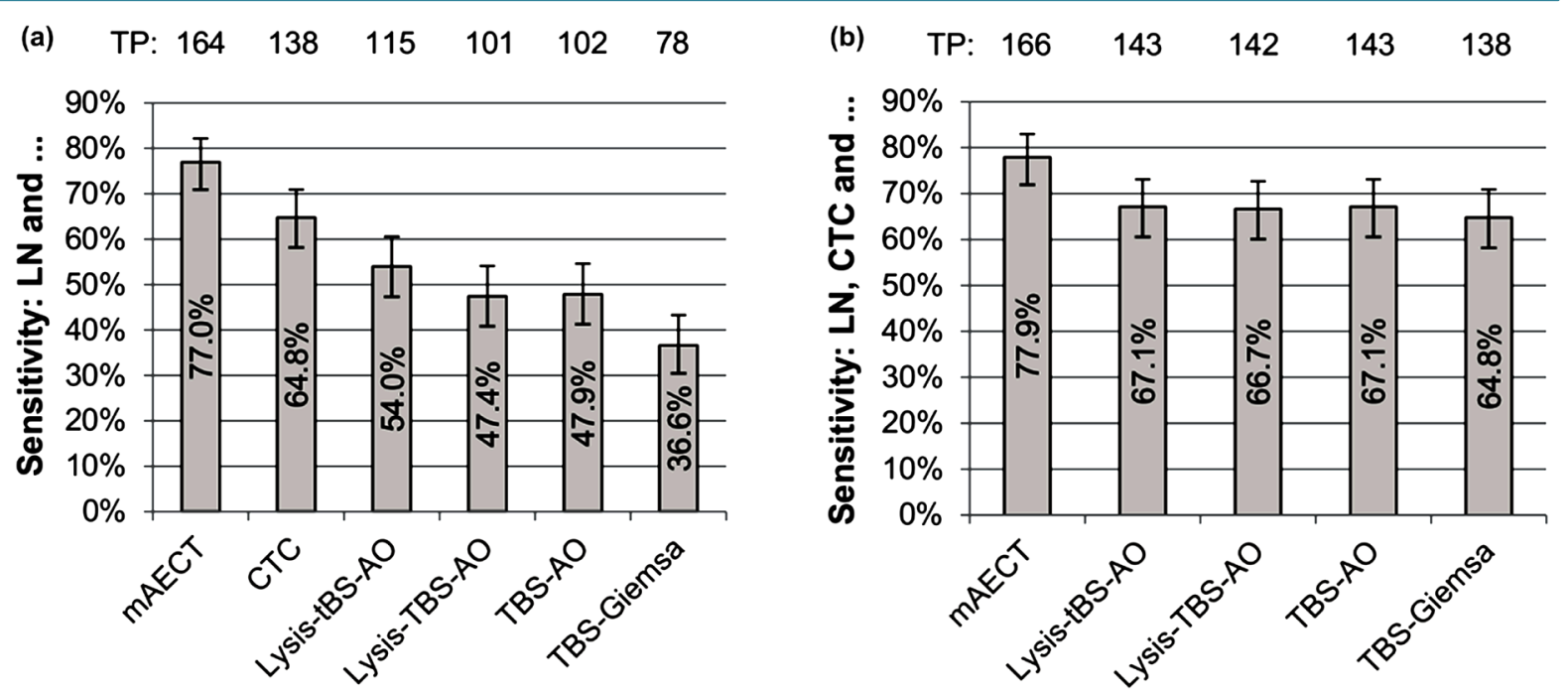

Figure 4. Sensitivity of diagnostic algorithms that include examination of lymph node aspirate in addition to other methods detecting HAT cases. (a) Diagnostic algorithms that include examination of lymph node (LN) aspirate in addition to one of the tests shown along the X axis. (b) Diagnostic algorithms that include examination of lymph node aspirate as well as capillary tube centrifugation (CTC) in addition to one of the tests shown along the X axis. The result of an algorithm is positive if one or several tests give a positive result. The number of HAT cases correctly identified with each algorithm (TP: true positives) is shown above the graphs. mAECT: miniature anion exchange centrifugation technique; Lysis-tBS-AO: thin blood smear $(20 \mu \mathrm{l})$ prepared after red blood cell lysis and concentration, stained with acridine orange and examined by fluorescence microscopy; Lysis-TBS-AO: thick blood smear $(20 \mu \mathrm{l})$ prepared after red blood cell lysis and concentration, stained with acridine orange and examined by fluorescence microscopy; TBS-AO: thick blood smear $(5 \mu \mathrm{l})$ stained with acridine orange and examined by fluorescence microscopy; TBS-Giemsa: thick blood smear ( $5 \mu 1)$ stained with Giemsa and examined by bright field microscopy. Error bars indicate $95 \%$ confidence intervals.

Processing blood samples using the RBC lysis and concentration procedure prior to preparing thin smears and staining them with acridine orange results in a further increase in sensitivity, which then becomes more than 5 times higher than thick smears stained with Giemsa. Although the lysis and concentration procedure requires a centrifugation step and is therefore more difficult to deploy than microscopy of whole blood, it can be an alternative to CTC or even mAECT, with the added value of long-term storage of the blood smears stained with acridine orange. In addition, the new methods described here do not require a cold chain, unlike other tests such as mAECT.

A slightly lower specificity was observed with CTC and mAECT when compared with the other methods. This could have been due to cases of HAT with low parasitaemia being missed during enrolment with routine diagnostic procedures, which were later found to be positive when re-tested with CTC and mAECT. The seemingly lower specificity of CTC and mAECT is therefore most likely associated to the design of this study, which involves two independent testing of samples, rather than to actual false positive results.

Fluorescence microscopy has previously been reported to improve detection of trypanosomes [22] [23]. However, the sophisticated and costly infrastructure required has until now been a hindrance to its application in clinical settings where HAT is endemic. New-generation LED fluorescence microscopes, such as the Primo Star iLED used in the present study, are much more affordable and simpler to use than conventional fluorescence microscopes, do not need a dark room, and consume very little energy, which enables them to be powered using a battery or a small solar panel.

We have recently shown that the time needed to find trypanosomes by fluorescence microscopic examination of acridine orange stained smears is significantly shorter than with bright field microscopy of Giemsa stained slides [16]. We also observe that in contrast to the white background that is typical in bright field microscopy, the dark background observed with fluorescence examination is less tiring for the reader, who can therefore comfortably scan a larger number of slides.

Acridine orange also has a practical, time-saving advantage over Giemsa. While most protocols for Giemsa staining require incubation for 20 to 50 minutes, acridine orange staining takes 3 minutes only, which allows for a larger number of microscopy slides to be processed in the same period of time. Similar observations have been 
made in studies on malaria diagnosis, in which LED FM was found to be four times faster than conventional light microscopy [24]. The cost of the reagents used to stain with acridine orange is equivalent to that of the ones routinely used for Giemsa staining, which makes the overall running cost per test lower when acridine orange is used, since less staff time is required. A cost-effectiveness study should, however, be conducted to formally compare various diagnostic algorithms.

With a steady decrease in the prevalence of HAT in most endemic countries, national control programmes are increasingly integrating control activities in the general health system, rather than relying on specialized health facilities and mobile teams that are dedicated to HAT. In this context, fluorescence microscopy can play a significant role in improving diagnosis at the primary healthcare level where, quite often, the only parasitological method available for case detection from blood samples is examination of Giemsa stained thick smears. Similarly, these methods will be very useful in settings where a high prevalence of microfilaria hinders the use of the CTC technique. In such settings, replacing the current method with fluorescence microscopy of thick smears stained with acridine orange could easily be achieved with minimal equipment and training investment, and would be expected to result in a 3 -fold higher case detection rate.

The procedures described here can have wider applications in diagnosis of other human and livestock diseases that rely on microscopic demonstration of parasites in blood, including malaria, leishmaniasis, Chagas disease, anaplasmosis, babesiosis and theileriosis among others. Indeed, recent studies in Gabon have demonstrated that LED FM can have a sensitivity of up to $99.1 \%$ in the detection of malaria parasites in blood [24].

\section{Acknowledgements}

We would like to thank the Swiss Tropical and Public Health Institute (Swiss TPH) for their help in developing the study protocol and associated documents, as well as a panel of experts who reviewed and suggested improvements to the study protocol. We also thank Didier Kalemwa (Swiss TPH) for monitoring the study, and Beatrice Gordis (FIND) for proof-reading the manuscript. This work was supported through FIND by the Bill and Melinda Gates Foundation and the Department for International Development (DFID) of the United Kingdom.

\section{References}

[1] WHO (2013) Control and Surveillance of Human African Trypanosomiasis. Report of a WHO Expert Committee. World Health Organization Technical Report Series, 984, 1-250.

[2] Simarro, P.P., Diarra, A., Ruiz Postigo, J.A., Franco, J.R. and Jannin, J.G. (2011) The Human African Trypanosomiasis Control and Surveillance Programme of the World Health Organization 2000-2009: The Way Forward. PLoS Neglected Tropical Diseases, 5, e1007. http://dx.doi.org/10.1371/journal.pntd.0001007

[3] WHO (2013) Trypanosomiasis, Human African (Sleeping Sickness) http://www.who.int/mediacentre/factsheets/fs259/en/

[4] Alirol, E., Shrumpf, D., Heradi, J.A., Riedel, A., de, P.C., Quere, M. and Chappuis, F. (2013) Nifurtimox-Eflornithine Combination Therapy (NECT) for Second-Stage Gambiense Human African Trypanosomiasis: MSF Experience in the Democratic Republic of the Congo. Clinical Infectious Diseases, 56, 195-203.

[5] Kennedy, P.G. (2008) Diagnosing Central Nervous System Trypanosomiasis: Two Stage or Not to Stage? Transactions of the Royal Society of Tropical Medicine and Hygiene, 102, 306-307.

[6] Kennedy, P.G. (2006) Diagnostic and Neuropathogenesis Issues in Human African Trypanosomiasis. International Journal for Parasitology, 36, 505-512.

[7] Brun, R. and Blum, J. (2012) Human African Trypanosomiasis. Infectious Disease Clinics of North America, 26, 261273.

[8] Lejon, V., Roger, I., Mumba, N.D., Menten, J., Robays, J., N'Siesi, F.X., Bisser, S., Boelaert, M. and Büscher, P. (2008) Novel Markers for Treatment Outcome in Late-Stage Trypanosoma brucei gambiense Trypanosomiasis. Clinical Infectious Diseases, 47, 15-22. http://dx.doi.org/10.1086/588668

[9] Lejon, V. and Büscher, P. (2005) Review Article: Cerebrospinal Fluid in Human African Trypanosomiasis: A Key to Diagnosis, Therapeutic Decision and Post-Treatment Follow-Up. Tropical Medicine \& International Health, 10, $395-$ 403.

[10] Van Nieuwenhove, S., Betu-Ku-Mesu, V.K., Diabakana, P.M., Declercq, J. and Bilenge, C.M. (2001) Sleeping Sickness Resurgence in the DRC: The Past Decade. Tropical Medicine \& International Health, 6, 335-341. 
[11] Simarro, P.P., Jannin, J. and Cattand, P. (2008) Eliminating Human African Trypanosomiasis: Where Do We Stand and What Comes Next? PLoS Medicine, 5, e55. http://dx.doi.org/10.1371/journal.pmed.0050055

[12] Franco, J.R., Simarro, P.P., Diarra, A., Ruiz-Postigo, J.A. and Jannin, J.G. (2012) The Human African Trypanosomiasis Specimen Biobank: A Necessary Tool to Support Research of New Diagnostics. PLoS Neglected Tropical Diseases, 6, e1571. http://dx.doi.org/10.1371/journal.pntd.0001571

[13] Büscher, P., Mumba, N.D., Kabore, J., Lejon, V., Robays, J., Jamonneau, V., Bebronne, N., Van der Veken, W. and Biéler, S. (2009) Improved Models of Mini Anion Exchange Centrifugation Technique (mAECT) and Modified Single Centrifugation (MSC) for Sleeping Sickness Diagnosis and Staging. PLoS Neglected Tropical Diseases, 3, e471. http://dx.doi.org/10.1371/journal.pntd.0000471

[14] Matovu, E., Mugasa, C.M., Ekangu, R.A., Deborggraeve, S., Lubega, G.W., Laurent, T., Schoone, G.J., Schallig, H.D. and Büscher, P. (2010) Phase II Evaluation of Sensitivity and Specificity of PCR and NASBA Followed by Oligochromatography for Diagnosis of Human African Trypanosomiasis in Clinical Samples from D.R. Congo and Uganda. PLoS Neglected Tropical Diseases, 4, e737. http://dx.doi.org/10.1371/journal.pntd.0000737

[15] Ndung'u, J.M., Bieler, S. and Roscigno, G. (2010) "Piggy-Backing" on Diagnostic Platforms Brings Hope to Neglected Diseases: The Case of Sleeping Sickness. PLoS Neglected Tropical Diseases, 4, e715. http://dx.doi.org/10.1371/journal.pntd.0000715

[16] Biéler, S., Matovu, E., Mitashi, P., Ssewannyana, E., Bi Shamamba, S.K., Bessell, P.R. and Ndung’u, J.M. (2012) Improved Detection of Trypanosoma brucei by Lysis of Red Blood Cells, Concentration and LED Fluorescence Microscopy. Acta Tropica, 121, 135-140. http://dx.doi.org/10.1016/j.actatropica.2011.10.016

[17] Chappuis, F., Loutan, L., Simarro, P., Lejon, V. and Büscher, P. (2005) Options for Field Diagnosis of Human African Trypanosomiasis. Clinical Microbiology Reviews, 18, 133-146. http://dx.doi.org/10.1128/CMR.18.1.133-146.2005

[18] Mitashi, P., Hasker, E., Lejon, V., Kande, V., Muyembe, J.J., Lutumba, P. and Boelaert, M. (2012) Human African Trypanosomiasis Diagnosis in First-Line Health Services of Endemic Countries, a Systematic Review. PLOS Neglected Tropical Diseases, 6, e1919.

[19] Agresti, A. and Coul, B.A. (1998) Approximate Is Better than "Exact" for Interval Estimation of Binomial Proportions. American Statistician, 52, 119-126. http://dx.doi.org/10.2307/2685469

[20] Crawley, M.J. (2007) The R Book. John Wiley \& Sons, Chichester. http://dx.doi.org/10.1002/9780470515075

[21] R Development Core Team (2008) R: A Language and Environment for Statistical Computing. R Foundation for Statistical Computing, Vienna.

[22] Hansen, D.W., Hunter, D.T., Richards, D.F. and Allred, L. (1970) Acridine Orange in the Staining of Blood Parasites. Journal of Parasitology, 56, 386-387. http://dx.doi.org/10.2307/3277684

[23] Lejon, V., Ameel, V., Brandt, J., Van den Bossche, P. and Büscher, P. (2003) Development of a Direct Immunofluorescence Test (DIFAT) for Parasitological Diagnosis of African Trypanosomosis. In: Wgzo, J., Ed., International Scientific Council for Trypanosomiasis Research \& Control (ISCTRC), Twenty Seventh Meeting, Pretoria, Organization of African Unity (OAU), Nairobi, Scientific and Technical Research Council (STRC), 2005, 185-189.

[24] Lenz, D., Kremsner, P.G., Lell, B., Biallas, B., Boettcher, M., Mordmüller, B. and Adegnika, A.A. (2011) Assessment of LED Fluorescence Microscopy for the Diagnosis of Plasmodium falciparum Infections in Gabon. Malaria Journal, 10, 194. http://dx.doi.org/10.1186/1475-2875-10-194 